\title{
PD-1 Directed Probody CX-188
}

National Cancer Institute

\section{Source}

National Cancer Institute. PD-1 Directed Probody CX-188. NCI Thesaurus. Code

C160716.

A probody composed of a monoclonal antibody directed against the negative immunoregulatory human cell surface receptor, programmed cell death protein 1 (PD-1; PDCD1; CD279), linked to a proprietary masking peptide that covers the active antigen binding site of the antibody through a protease-cleavable linker, with potential immune checkpoint inhibitory and antineoplastic activities. Upon administration of PD-1-directed probody CX-188, the masking peptide is cleaved by tumor-associated proteases within the tumor microenvironment (TME). Protease-mediated removal of the linker enables binding of the unmasked monoclonal antibody moiety to PD-1, thereby disrupting PD-1 signaling. This may restore immune function through the activation of T-cells and T-cellmediated immune responses against tumor cells. PD-1, a transmembrane protein in the immunog lobulin superfamily (IgSF) expressed on T-cells, functions as an immune checkpoint that negatively regulates T-cell activation and effector function when activated by its ligands programmed cell death-1 ligand 1 (PD-L1) or 2 (PD-L2); it plays an important role in tumor evasion from host immunity. Tumor-associated proteases are present in high concentrations and aberrantly activated in the TME. 